\title{
An $x$-ray microscopic study of the vasa vasorum of the normal human aortic arch
}

\author{
J O H N A. CLARKE \\ From the Department of Anatomy, University of Glasgow
}

Descriptions of the vasa vasorum of the aortic wall vary. The main papers are concerned with the intramural vasculature of the descending aorta, little reference being made to the aortic arch. The first observation on the vasa vasorum of the aortic wall is attributed to Willis (Haller, 1757).

In a review of the literature, Ramsey (1936), recording the descriptions of the principal nineteenth century investigators (Risse, 1853 ; Gimbert, 1865 ; Plotnikow, 1884), showed that interpretations of injected and routine histological specimens of the aortic wall differed on the depth the intramural vessels penetrated.

From an examination of animal aortae injected with India ink, Woodruff (1926) concluded that the aortic vasa arose from collateral branches of the aorta, to be distributed to the adventitia and inner media.

Robertson (1929), injecting human thoracic aortae and animal aortae with coloured cellulose, demonstrated networks of adventitial vessels and concluded that the outer third of the media in the human aorta was vascularized.

By injecting the aortic wall with Higgins ink and clearing in glycerine or by the Spalteholz method, Winternitz, Thomas, and LeCompte (1938) revealed vascular patterns in the outer media of the human and animal arterial wall and demonstrated intimal vascularization in atheromatous plaques.

Geiringer (1951), from an examination of frozen sections of human aorta, concluded that the normal aortic intima was avascular, only acquiring a secondary blood supply if it attained a critical thickness of $0.5 \mathrm{~mm}$.

The introduction of microradiographic techniques in arteriography (Barclay, 1947) permitted the study of the microcirculation without routine histological preparation of tissues. de Sousa and Alvares (1960) and Nylander and Olerud (1960), employing microradiography, demonstrated the vasa vasorum of the aorta and inferior vena cava in the dog, revealing the adventitial arteriolar plexus and the patterns of distribution to the inner layers of the vessel wall.

This work is concerned with a description of the vasa vasorum in the normal human aortic arch, $\vec{c}$ using the Coslett-Nixon $x$-ray projection microscope. This enables a study of the pattern and distribution of the vasa vasorum in freshly injected specimens to be made, in contrast to the routine $\vec{C}$ histological and clearing techniques of previous $\stackrel{\leftrightarrow}{\mathcal{G}}$ investigators.

\section{MATERIAL AND METHODS}

Fifty normal post-mortem aortic arches were examined within eight hours of death from hearts in the age group 15-80 years.

The microcirculation in the aortic wall was demonstrated by injecting micropaque at physiological pressures, the arterial side through the innominate and bronchial arteries, the venous side through the bronchial veins.

$X$-ray projection micrographs of full thickness aortic wall and $1 \mathrm{~mm}$. thick sections were taken on $\tilde{x}$ Ilford contrasty plates with an exposure time of $10 \dot{\sigma}$ minutes. The microscope was operated at $15 \mathrm{kV}$ and 3 $40 \mathrm{~mA}$ with a copper target providing the $x$-radiation.

\section{OBSERVATIONS}

From an examination of the micrographs it was concluded that the arterial supply to the wall of $N$ the aortic arch originated from the ostium of the innominate artery and the terminal branches of the bronchial arteries.

It was found that tightly coiled arterioles, 80 to $100 \mu$ in diameter, originated from the ostium of 0 the innominate artery to be distributed to the adventitia of the summit of the aortic arch (Figs. 1 ? and 2) while the sides and concave aspect of the $\frac{T}{0}$ arch were supplied by sinuous adventitial arterioles, 60 to $80 \mu$ in diameter, from the bronchial arteries (Figs. 3 and 4).

From the adventitial plexus, arterioles 60 to $80 \mu$ 응 in diameter penetrated the deep layers of the $\delta$ 
An $x$-ray microscopic study of the vasa vasorum of the normal human aortic arch

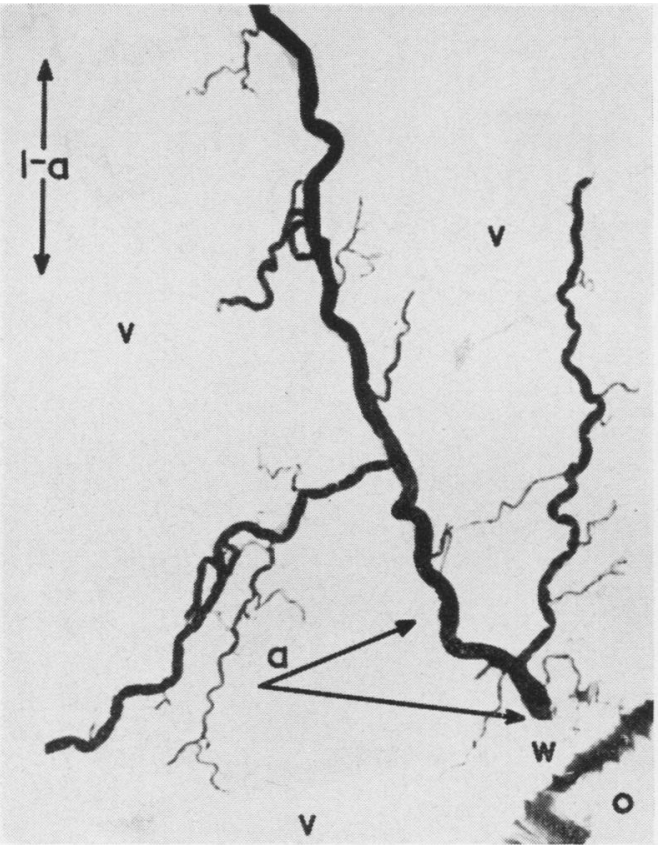

Fig. 1

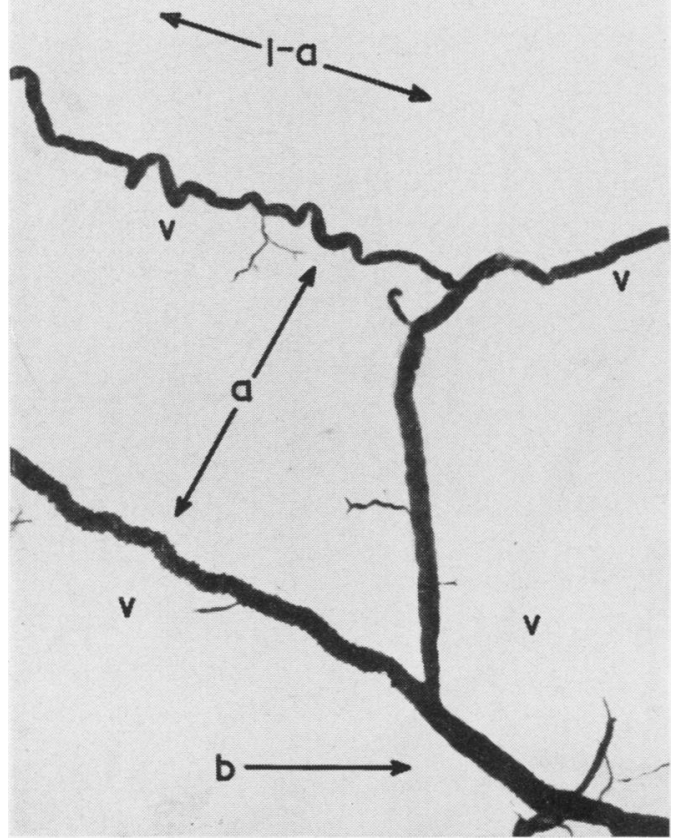

FIG. 3

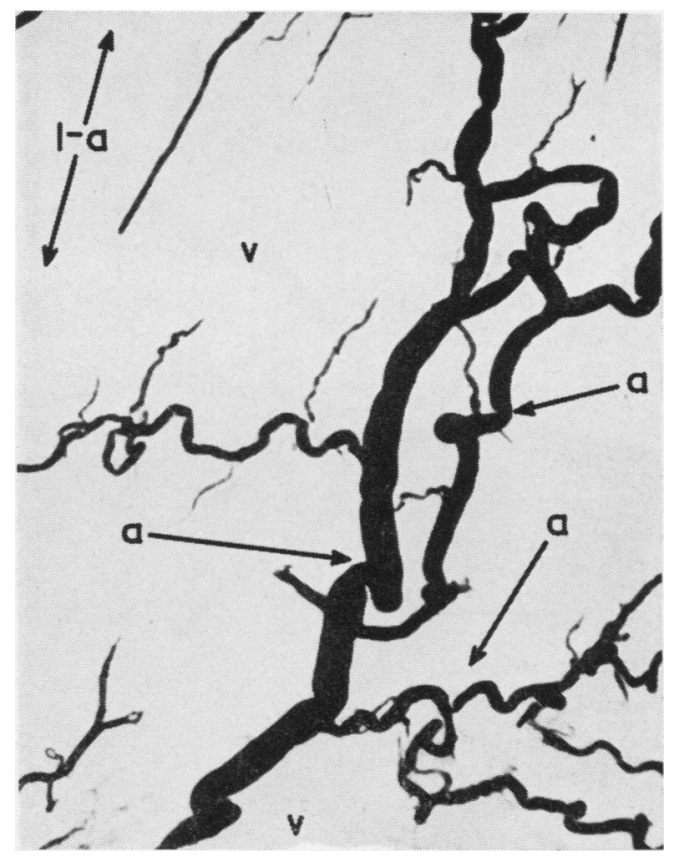

FIG. 2

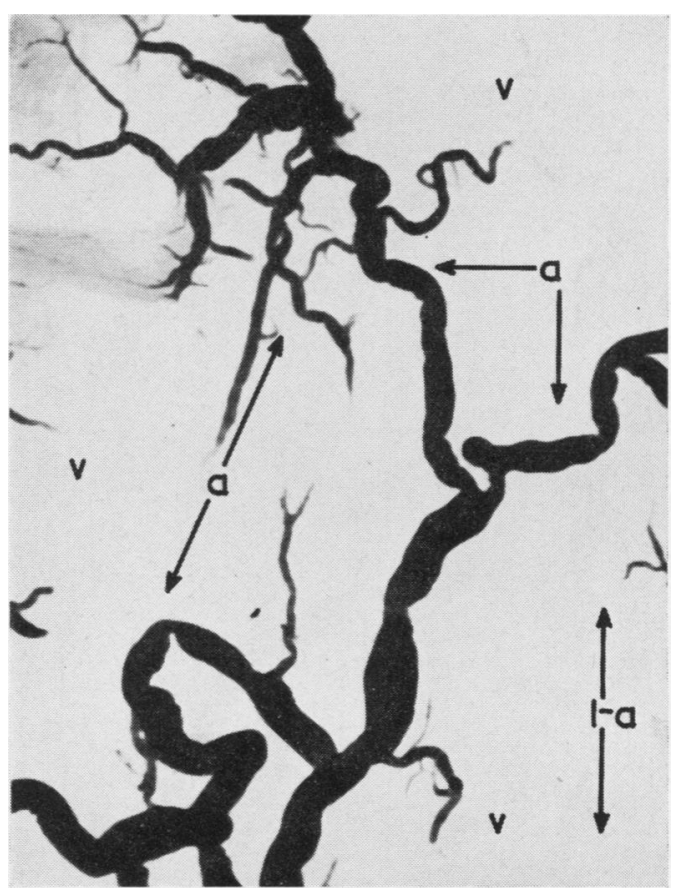

FIg. 4

For explanation of Figs 1 to 12 see p. 80. 


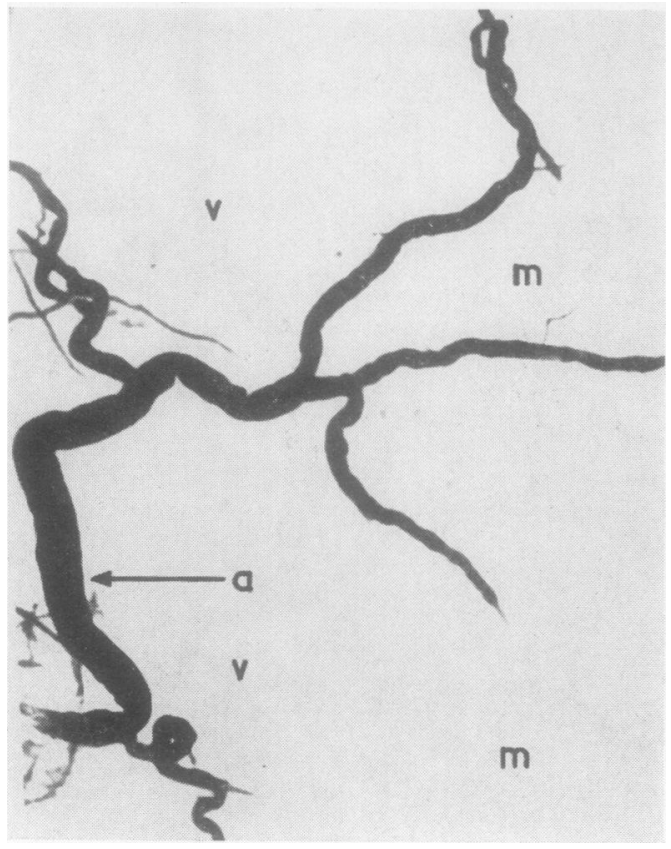

FIG. 5

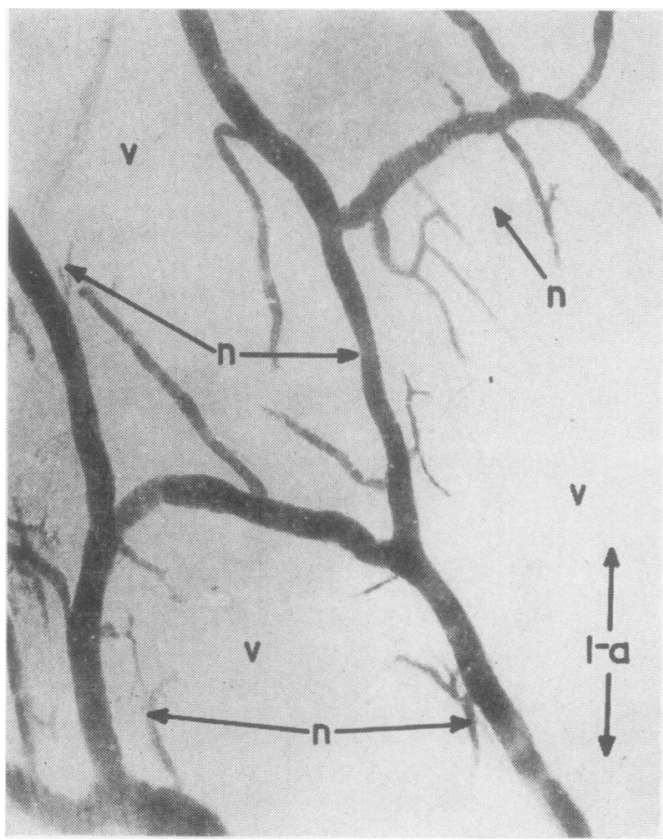

FIG. 7

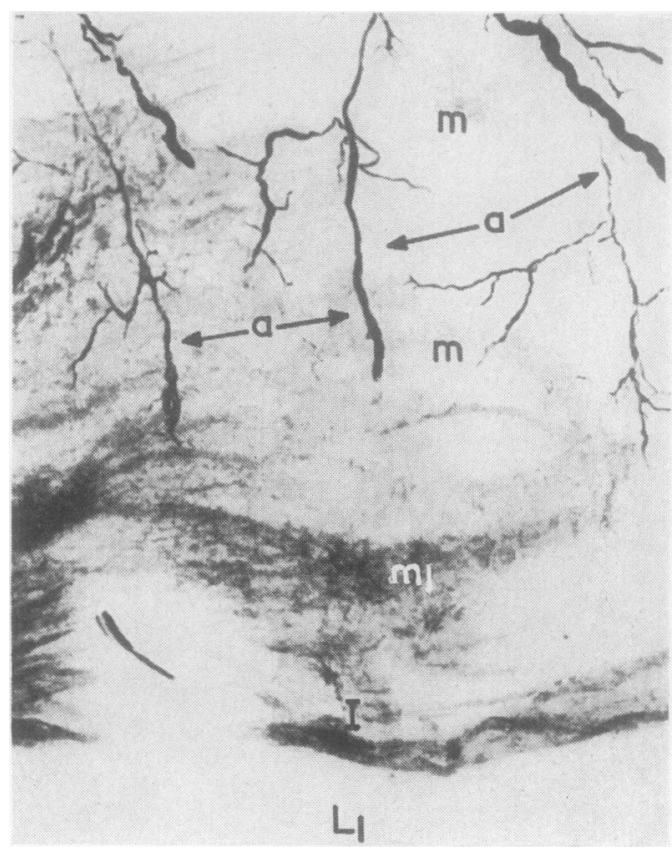

FIG. 6

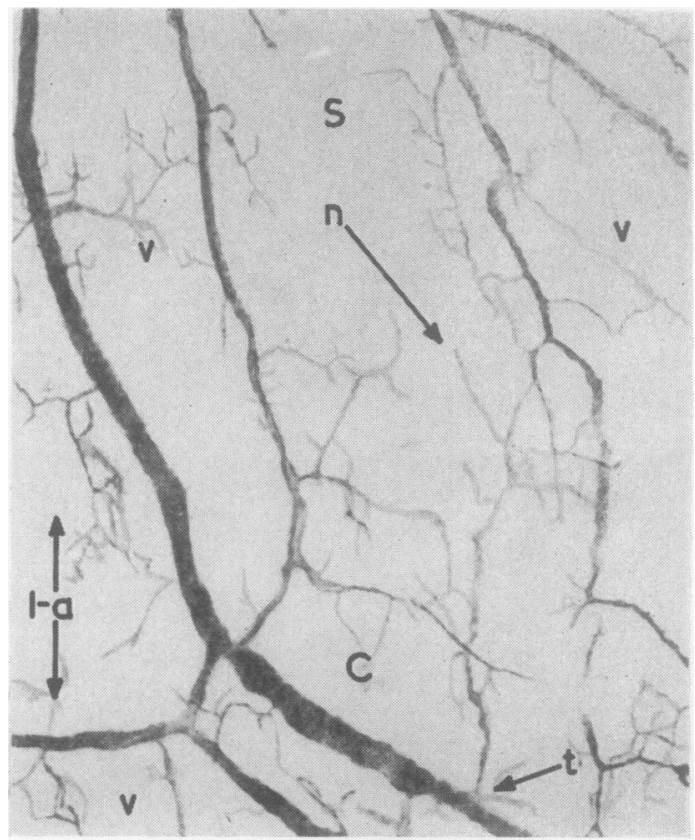

Frg. 8 


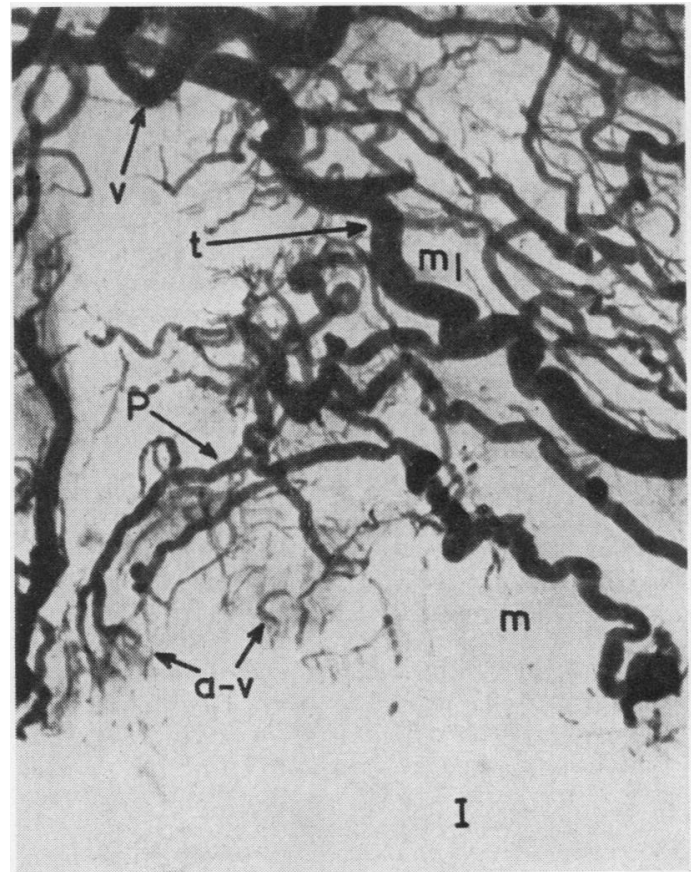

FIG. 9

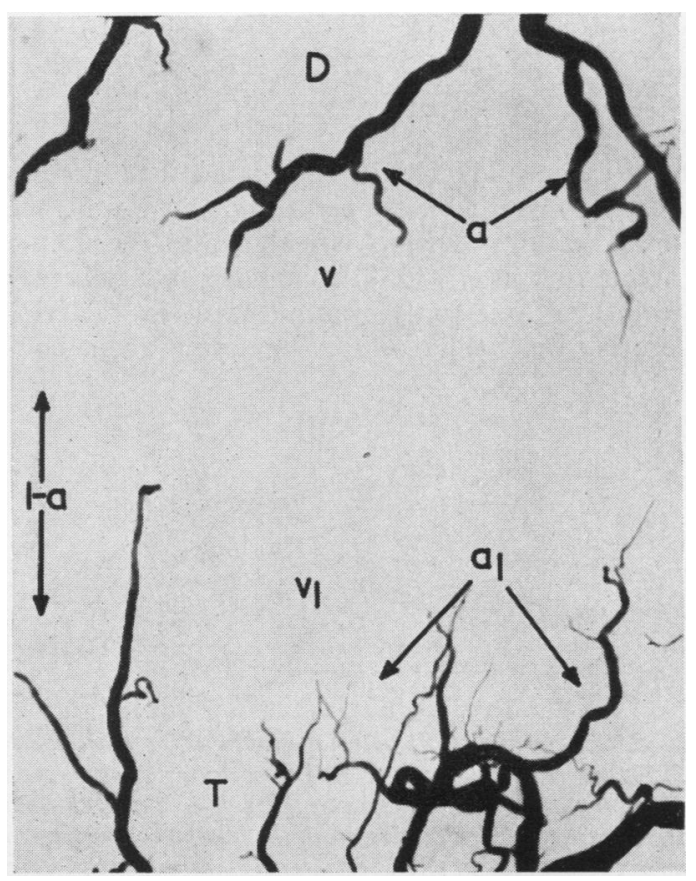

FIG. 11
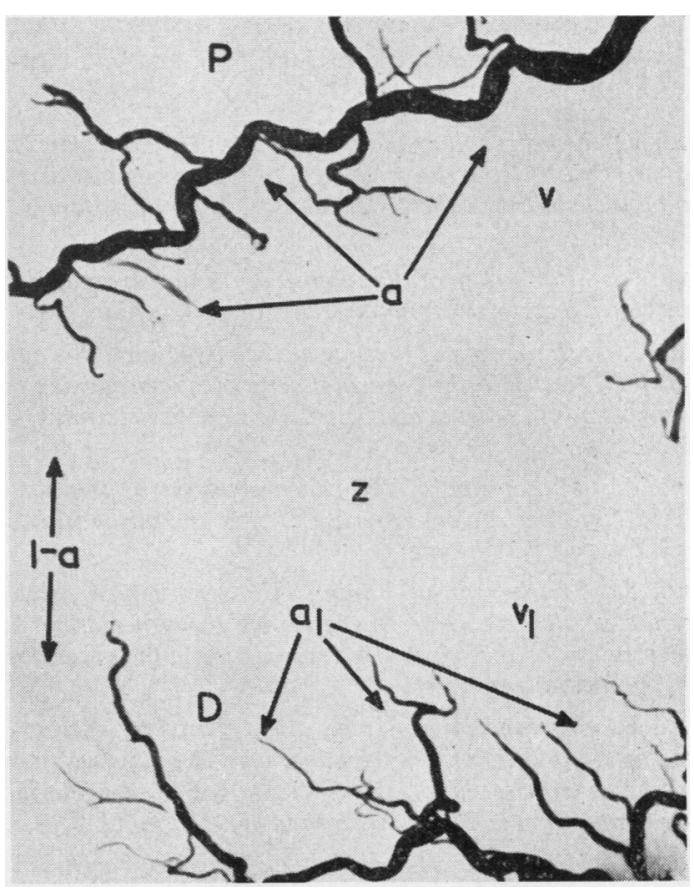

FIG. 10

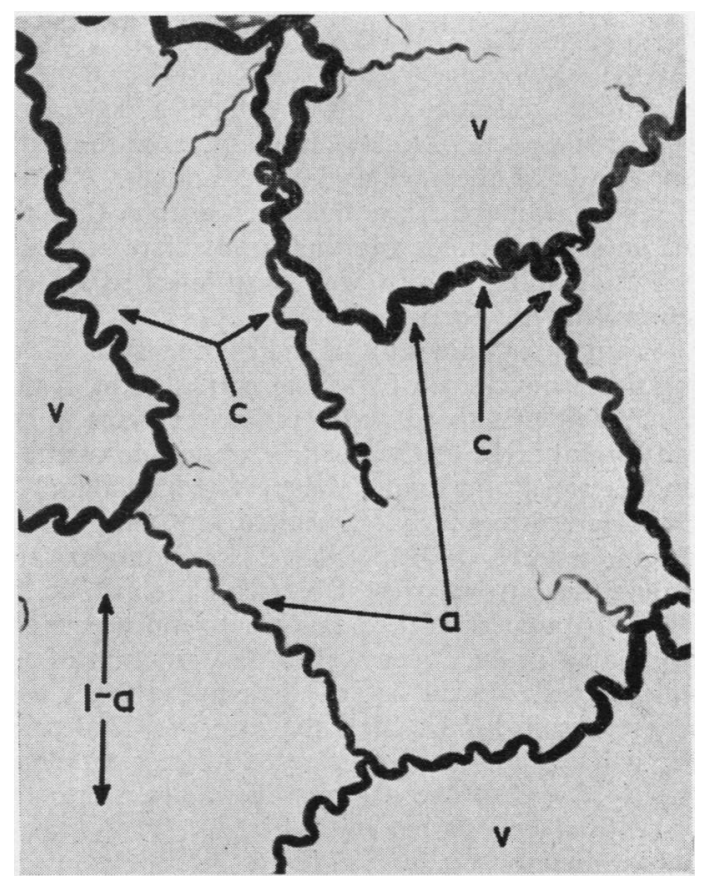

FIG. 12 


\section{EXPLANATION OF PLATES}

All figures, except Figs. 5, 6, and 9, are of full thickness aortic wall. Long axis of aorta (1-a).

FIG. 1. Micrograph of the aortic arch. Note the arteriole (a) originating from the ostium $(o)$ of the innominate artery to be distributed to the adventitia (v). Wall of ostium (w), $\times 20$.

FIG. 2. Micrograph of the summit of the aortic arch. Note the coiled arterioles $(a)$ in the adventitia $(v), \times 45$.

FIG. 3. Micrograph of the side of the aortic arch. Note the terminal branch of the bronchial artery (b) approaching the adventitia $(v)$ to divide and distribute sinuous arterioles $(a)$, $\times 30$.

FIG. 4. Micrograph of the concave aspect of the aortic arch. Note the sinuous arterioles $(a)$ in the adventitia (v), $\times 70$.

FIG. 5. Micrograph of $1 \mathrm{~mm}$. thick longitudinal section of the aortic arch. Note the arteriole (a) penetrating the deep layers of the adventitia $(v)$ to divide in the outer third of the media $(\mathrm{m}), \times 50$.

FIG. 6. Micrograph of $1 \mathrm{~mm}$. thi-k transverse section of the aortic arch. Note the terminal arterial distribution (a) in the outer two-thirds of the media $(m)$. Inner third of the media $\left(m_{1}\right)$ is avascular. Intima $(I)$. Lumen $\left(L_{1}\right), \times 50$.

FIG. 7. Micrograph of the summit of the aortic arch. Note the sparse irregular venous network $(n)$ in the adventitia (v), $\times 30$.

adventitia, bifurcated, and formed a secondary plexus of vessels 10 to $20 \mu$ in diameter in the outer two-thirds of the media (Figs. 5 and 6).

It was evident from the micrographs that the adventitial arterioles and their immediate branches showed coiling, which was most marked on the summit of the aortic arch (Fig. 2).

From an examination of the micrographs showing the venous side of the microcirculation, it was concluded that there was a plexus of veins in the adventitial layer of the aortic arch, predominantly on the sides and concave aspect (Figs. 7 and 8). Arranged irregularly, these adventitial venous channels were 100 to $140 \mu$ in diameter and drained into tributaries of bronchial veins (Fig. 8).

The tributaries of the adventitial venous network originated in the aortic wall at the junction of the intimal and medial layers, thus appearing closer to the aortic lumen than the arteries. Coalescing in an irregular pattern, these tributaries traversed the thickness of the aortic wall to drain into the adventitial venous network (Fig. 9).

No injection medium entered the intima in any specimen examined.
FIG. 8. Micrograph of the side $(S)$ and concave aspect $(C)$ of the aortic arch. Note the dense venous network $(n)$ in the adventitia (v), draining into tributary $(t)$ of the bronchial veins, $\times 25$.

FIG. 9. Micrograph of $1 \mathrm{~mm}$. thick longitudinal section of the aortic arch. Note the origin of the venous plexus $(p)$ in the inner third of the media $(m)$ draining into tributaries $(t)$ of the adventitial veins ( $v)$. Terminal arteriovenous anastomoses $(a-v)$. Outer two-thirds of media $\left(m_{1}\right)$. Intima $(I), \times 30$.

FIG. 10. Micrograph of the proximal end of the aortic arch $(P)$ and the distal end of the ascending aorta $(D)$. Note the arterioles $(a)$ in the adventitia $(v)$ of the aortic arch, and the arterioles $\left(a_{1}\right)$ in the adventitia $\left(v_{1}\right)$ of the ascending aorta. Avascular zone $(z), \times 20$.

FIG. 11. Micrograph of the distal end of the aortic arch $(D)$ and the proximal end of the thoracic aorta $(T)$. Note the arterioles $(a)$ in the adventitia of the aortic arch $(v)$ and the arterioles $\left(a_{1}\right)$ in the adventitia $\left(v_{1}\right)$ of the thoracic aorta, $\times 20$.

FIG. 12. Micrograph of the summit of the aortic arch, aged 65 years. Note the increased coiling $(c)$ of the arterioles $(a)$ in the adventitia $(v), \times 20$.

\section{DISCUSSION}

The present study has demonstrated that there is an extensive vascular supply to the aortic wall, the general features of which are shown in Fig. 13.

Winternitz et al. (1938) concluded that, whereas the intima was only vascularized in 'arteriosclerosis', the media of the ascending aorta con-

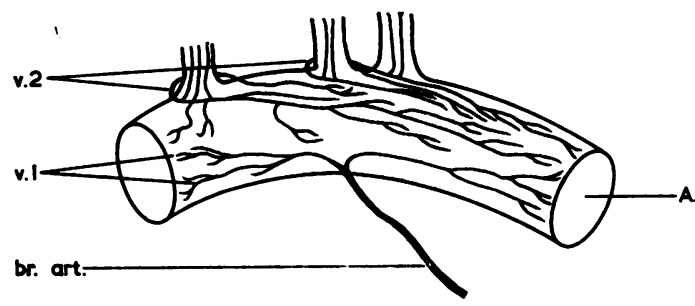

FIG. 13. Diagram of the arterial supply to the aortic arch (A). Note terminal branch of the bronchial artery (br. art.) distributing arterial vasa (v.1) to the side of the arch, and arterial vasa (v.2) originating from the innominate artery to supply the summit of the arch. Occasionally arterial vasa arise from the left common carotid artery (v.2). 
tained 'large sinusoids' which were venous vasa. Woerner (1959) examined serially cut $400 \mu$ sections of the ascending aorta and reported 'a vascular area in the inner half of the wall' in one specimen. In this series it was confirmed that the intima was avascular.

The coiling of the adventitial arterioles was interpreted as a defence mechanism against excessive stretch during systole.

Particular attention was paid to the arrangement of the arterial vasa at the proximal and distal ends of the aortic arch (Figs. 10 and 11). The arterial vasa in the adventitia of the ascending aorta have been shown to terminate $1 \mathrm{~cm}$. proximal to the origin of the innominate artery (Clarke, 1964), and the arterial vasa of the thoracic aorta to arise segmentally from the intercostal arteries (unpublished observation). No anastomoses were observed between the arterial vasa of the arch and the ascending and thoracic aorta respectively, and it is suggested that these poorly vascularized areas are potential weaknesses in the aortic wall.

The predominance of the adventitial veins on the sides and the concavity of the aortic arch indicates a specific function. It is conceivable that the site of these veins protects them from the occlusive effect of the systolic thrust, which is maximal on the summit of the aortic arch, and permits a continuous drainage.

It is evident from the micrographs that tortuous vessels 25 to $30 \mu$ in diameter lie at the junction of the intima and inner third of the media (Fig. 9). These were only found after the venous side of the circulation had been injected. It is suggested that they correspond to the venous vasa described by Winternitz.

It was concluded that since small arterioles and capillaries, $10 \mu$ to $20 \mu$ in diameter, could be identified in the middle layer of the media, the terminal arteriovenous anastomoses occurred in the inner third of the media (Fig. 9). With age, although there was no evidence of an increase in arterial vascularity, the adventitial arterioles were more tightly coiled in specimens over 50 years of age (Fig. 12).

\section{SUMMARY}

The arterial and venous sides of the microcirculation in the normal human aortic arch have been shown.

The arteries originate from the ostium of the innominate artery and the terminal branches of the bronchial arteries. They are distributed to the adventitia and outer two-thirds of the media.

The veins originate in the inner third of the media and drain through the artery wall into irregular adventitial venous plexuses, which are tributaries of the bronchial veins.

The terminal arteriovenous anastomosis occurs in the inner third of the media.

No injection medium entered the intima.

I am grateful to Professor G. M. Wyburn for his interest in this study.

I am also grateful to Professor D. F. Cappell, Pathology Department, Glasgow University, whose co-operation enabled this work to be done.

I am indebted to the Medical Research Council whose aid allowed the purchase of the $x$-ray microscope.

\section{REFERENCES}

Barclay, A. E. (1947). Micro-arteriography. Brit. J. Radiol., $20,394$. Clarke, J. A. (1964). An X-ray microscopic study of the vasa vasorum of the normal human ascending aorta. Brit. Heart J., 27, 99.

Geiringer, E. '(1951). Intimal vascularization and atherosclerosis. J. Path. Bact., 63, 201.

Gimbert, J. L. (1865). Structure et Texture des Artères. Thèse de Paris,

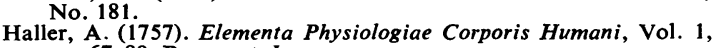
pp. 67-89. Bousquet, Lausanne,

Nylander, G., and Olerud, S. (1960). The distribution of the vasa vasorum in the abdominal aorta and the vena cava inferior in dogs. Angiology, 11, 522 .

Plotnikow, V. (1884). Untersuchungen über die Vasa Vasorum. Inaug.-Diss., Dorpat, 1884.

Ramsey, E. M. (1936). Studies in the pathology of vascular disease. I. Nutrition of the blood vessel wall. Review of the literature. Yale J. Biol. Med., 9, 13.

Risse, A. (1853). Observationes quaedam de arteriarum statu normali atque pathologico. Inaug. Dis., Regiomonti Pr., E. J. Dalkowski, 1853 .

Robertson, H. F. (1929). Vascularization of the thoracic aorta. Arch. Path., 8, 88i.

de Sousa, A., and Alvares, L. (1960). Microangiographic study of the vasa vasorum of the thoracic aorta. Radiology, 75, 91.

Winternitz, M. C., Thomas, R. M., and LeCompte, P. M. (1938). The Biology of Arteriosclerosis. Charles C. Thomas, Springfield, Illinois.

Woerner, C. A. (1959). Vasa vasorum of arteries, their demonstration and distribution. In The Arterial Wall, edited by A. I. Lansing, pp. 1-14. Williams and Wilkins, Baltimore.

pp. 1-14. Williams and Wilkins, Baltimore.
Woodruff, C. E. (1926). Studies on the vasa vasorum. Amer. J. Path., 2, 567 . 